Food Allergy: Molecular Basis and Clinical Practice 


\section{Chemical Immunology and Allergy}

Vol. 101

Series Editors

Johannes Ring Munich

Kurt Blaser Davos

Monique Capron Lille

Judah A. Denburg Hamilton, Ont.

Stephen T. Holgate southampton

Gianni Marone Naples

Hirohisa Saito Tokyo 


\section{Food Allergy: Molecular Basis and Clinical Practice}

Volume Editors

Motohiro Ebisawa Sagamihara

Barbara K. Ballmer-Weber zurich

Stefan Vieths Langen

Robert A. Wood Baltimore, Md.

19 figures, 4 in color, and 56 tables, 2015 
Chemical Immunology and Allergy

Formerly published as 'Progress in Allergy' (founded 1939),

continued 1990-2002 as 'Chemical Immunology'

Edited by Paul Kallós 1939-1988, Byron H. Waksman 1962-2002

Motohiro Ebisawa, MD, PhD

Department of Allergy

Clinical Research Center for Allergology and

Rheumatology

Sagamihara National Hospital

Sagamihara, Kanagawa, Japan

\section{Stefan Vieths, PhD}

Paul-Ehrlich-Institut

Langen, Germany

\author{
Barbara K. Ballmer-Weber, MD \\ Allergy Unit \\ Department of Dermatology \\ University Hospital Zurich \\ Zurich, Switzerland
}

\section{Robert A. Wood, MD}

Division of Pediatric Allergy and Immunology

Johns Hopkins University School of Medicine and

Department of International Health

Johns Hopkins Bloomberg School of Public Health

Baltimore, Md., USA

Bibliographic Indices. This publication is listed in bibliographic services, including Current Contents ${ }^{\circledR}$ and PubMed/MEDLINE.

Disclaimer. The statements, opinions and data contained in this publication are solely those of the individual authors and contributors and not of the publisher and the editor(s). The appearance of advertisements in the book is not a warranty, endorsement, or approval of the products or services advertised or of their effectiveness, quality or safety. The publisher and the editor(s) disclaim responsibility for any injury to persons or property resulting from any ideas, methods, instructions or products referred to in the content or advertisements.

Drug Dosage. The authors and the publisher have exerted every effort to ensure that drug selection and dosage set forth in this text are in accord with current recommendations and practice at the time of publication. However, in view of ongoing research, changes in government regulations, and the constant flow of information relating to drug therapy and drug reactions, the reader is urged to check the package insert for each drug for any change in indications and dosage and for added warnings and precautions. This is particularly important when the recommended agent is a new and/or infrequently employed drug.

All rights reserved. No part of this publication may be translated into other languages, reproduced or utilized in any form or by any means electronic or mechanical, including photocopying, recording, microcopying, or by any information storage and retrieval system, without permission in writing from the publisher.

(c) Copyright 2015 by S. Karger AG, P.O. Box, CH-4009 Basel (Switzerland)

www.karger.com

Printed in Germany on acid-free and non-aging paper (ISO 9706) by Kraft Druck GmbH, Ettlingen

ISSN 1660-2242

e-ISSN 1662-2898

ISBN 978-3-318-02340-4

e-ISBN 978-3-318-02341-1

\section{KARGER 125}




\section{Contents}

xv Preface

Ebisawa, M. (Sagamihara); Ballmer-Weber, B.K. (Zurich); Vieths, S. (Langen); Wood, R.A. (Baltimore, Md.)

\section{Background}

$1 \quad$ Historical Background, Definitions and Differential Diagnosis

Sampson, H.A. (New York, N.Y.)

1 Abstract

1 Historical Background

3 Definitions and Differential Diagnosis

5 Recent History and the Future

7 References

8 Immunological Basis of Food Allergy (IgE-Mediated, Non-IgE-Mediated, and Tolerance)

Kim, E.H.; Burks, W. (Chapel Hill, N.C.)

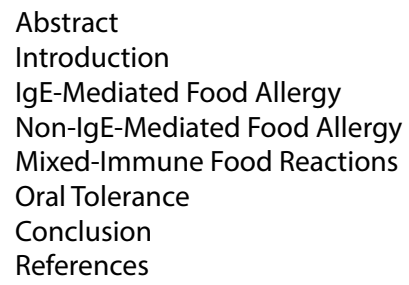

Food Allergens: Molecular and Immunological Aspects, Allergen Databases and Cross-Reactivity

Lorenz, A.-R.; Scheurer, S.; Vieths, S. (Langen)

18 Abstract

19 Molecular Features of Food Allergens

Allergen Families Allergen Databases

Class I Food Allergens/Class II Food Allergens

Class I Food Allergens

Class II Food Allergens

Resistance to Food Processing and Digestion

Thermal Stability of Allergens

Influence of Digestion on Allergic Reaction to Food Allergens

Influence of the Food Matrix on Digestion and Allergenicity of Food Allergens 
Immunological Features of Food Allergens

T Cell and B Cell Epitopes of Food Allergens

T Cell Epitopes

B Cell Epitopes

Cross-Reactivity

References

\section{Epidemiology: International Point of View, from Childhood to Adults, Food Allergens}

Wong, G.W.K. (Hong Kong)

Abstract

Epidemiology of Food Allergy

Reported Food Allergy and Epidemiology Study Methodology

Prevalence of Common Food Allergies

Changing Prevalence of Food Allergy

References

\section{Clinical Aspects}

\section{Food Allergy in Childhood (Infancy to School Age)}

Bergmann, M.M.; Eigenmann, P.A. (Geneva)

Abstract

Introduction

IgE-Mediated (Immediate-Type) Food Allergy

Food-Induced Urticaria and Anaphylaxis

Food-Dependent Exercise-Induced Anaphylaxis

Oral Allergy Syndrome

Mixed IgE and Non-IgE-Mediated Food Allergy

Atopic Dermatitis and Food Allergy

Eosinophilic Gastroenteropathies (Esophagitis, Gastritis, Enteritis, and Gastroenteritis)

Non-lgE-Mediated Food Allergy

Food Protein-Induced Enterocolitis Syndrome

Food Protein-Induced Proctocolitis

Food Protein-Induced Enteropathy

Heiner Syndrome (Milk-Induced Pulmonary Disease)

Conclusions

References

\section{Food Allergy in Adolescence and Adulthood}

Ballmer-Weber, B.K. (Zurich)

Abstract

Introduction

Food Allergies Due to Cross-Reaction with Inhalant Allergens

Birch Pollen-Mediated Food Allergy

Mugwort Pollen-Mediated Food Allergy

Grass, Ragweed, and Plane Pollen-Mediated Food Allergy

Other Cross-Reactive Food Allergies

Allergic Reactions to Foods May Already Occur upon First Consumption

Sensitization to Galactose-Alpha-1,3-Galactose and Allergy to Red Meat

Clinical Symptoms

Local Oral Symptoms

Warning Signs for Impending Systemic Reactions

Food Allergy-Induced Systemic Reactions

Differential Diagnosis of Food Allergy in Adults

Lactose Intolerance 


\title{
Diagnosis and Management
}

$59 \quad$ Hints for Diagnosis

Poulsen, L.K. (Copenhagen)

59 Abstract

59 The Purpose of the Diagnosis

59 Is It Food Allergy?

61 Which Is the Culprit Food?

62 Which Allergens Are Involved?

62 How Much Is Too Much?

63 Prerequisites for Diagnosis

63 The Diagnostic Process

66 Who Should Perform the Diagnosis?

66 Conclusions

66 References

68

(1)

\section{IgE-Related Examination in Food Allergy with Focus on Allergen Components}

Borres, M.P. (Uppsala); Sato, S.; Ebisawa, M. (Sagamihara)

\section{Abstract}

How IgE-Mediated Food Allergy Is Diagnosed

Component-Resolved Diagnostics

Development of a Test for Allergen Components

Heat Stability in Proteins

Species-Specific and Cross-Reactive Proteins

New Allergen Component Test: An Emerging Field of Interest

Temporal Development of Allergen Components

Singleplex versus Multiplex Testing

Future Perspectives

Skin Prick Test

Basophil Activation Test

Mechanisms of Basophil Activation

Histamine Release Test

Expression of the Basophil Activation Markers CD63 and CD203c

Correlation of the Skin Prick Test and the Basophil Activation Test with Oral Immunotherapy

References

79

\section{Non-IgE-Related Diagnostic Methods (LST, Patch Test)}

Matsumoto, K. (Tokyo)

\author{
Abstract \\ Introduction \\ Lymphocyte Stimulation Test \\ Peripheral Blood Mononuclear Cells \\ Antigens \\ Culture System \\ Measurements \\ Interpretation \\ Future Perspectives \\ Patch Test/Atopy Patch Test \\ Indication for Testing \\ Atopy Patch Test Methods
}




\section{Wood, R.A. (Baltimore, Md.)}

87

87

87

88

Abstract

Introduction Mild to Moderate Reactions

Anaphylaxis Adrenaline Adjunctive Therapies Observation Risk Minimisation Conclusions

References

Abstract

Introduction

Novel Treatment for Food Allergy

Sublingual Immunotherapy

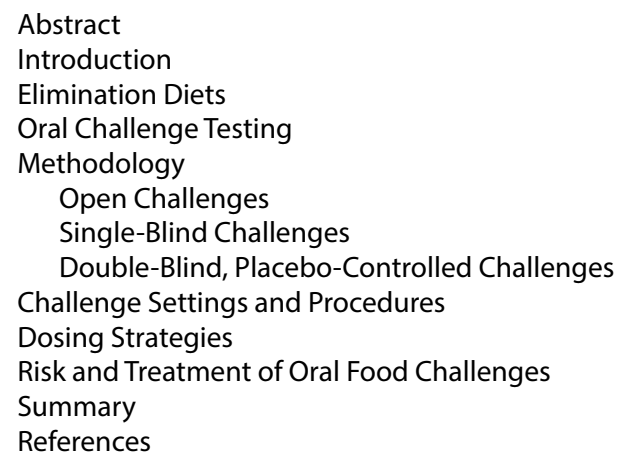

\section{Pharmacological Management of Acute Food-Allergic Reactions}

Richards, S.; Tang, M. (Melbourne, Vic.)

Pharmacological Management of IgE-Mediated, Food-Induced Acute Allergic Reactions

Subsequent Management of IgE-Mediated Food-Induced Allergic Reactions

Pharmacological Management of Non-IgE-Mediated Food-Induced Allergic Reactions

\section{Oral Immunotherapy and Potential Treatment}

Sato, S.; Yanagida, N.; Ebisawa, M. (Sagamihara)

Oral Immunotherapy for Food Allergy

Efficacy and Safety of Oral Immunotherapy

Factors Affecting Safety of Oral Immunotherapy

Mechanisms of and Immunologic Responses to Oral Immunotherapy

Clinical Use of Oral Immunotherapy

Desensitization and Permanent Tolerance

Optimal Dose and Duration of Oral Immunotherapy

Anti-IgE Monoclonal Antibodies (Omalizumab) for Food Allergy 
111 Epicutaneous Immunotherapy

112 Conclusion

112 Acknowledgments

112 References

\section{Allergen-Based Food Allergies}

\section{Cow's Milk Allergy in Children and Adults}

Fiocchi, A.; Dahdah, L. (Rome); Albarini, M.; Martelli, A. (Milan)

114 Abstract

114 Introduction

114 Epidemiology

115 Pathogenesis

115 Allergens

115 Clinical Manifestations

116 Immediate Reactions

116 Delayed Reactions

117 Diagnosis

117 Clinical Evaluation

117 Diagnostic Tests

117 IgE-Mediated CMA

117 Non-IgE-Mediated CMA

118 Elimination-Challenge Testing

118 Natural History

118 Treatment of Cow's Milk Allergy

118 Avoidance of Cow's Milk Protein

119 Alternative Formulae (Milk Substitutes)

119 Oral Immunotherapy

120 Follow-Up

121 A Very Common Clinical Case

122 Conclusions

122 References

124 Hen's Egg Allergy

Urisu, A.; Kondo, Y. (Nagoya); Tsuge, I. (Toyoake)

124 Abstract

124 Introduction

125 Symptoms

125 Allergenic Components of Egg White

126 Allergenic Components of Egg Yolk

126 Diagnosis

127 Natural History

128 Management and Therapy

128 Oral Immunotherapy to Egg Allergy

129 Clinical Case

129 Acknowledgments

129 References

131

$\begin{array}{ll}131 & \text { Abstract } \\ 131 & \text { Introduction } \\ 131 & \text { Epidemiology }\end{array}$ 
Clinical Reactions

Age of Presentation or First Reaction to Peanut and Tree Nuts

Co-Allergy to Peanut and Tree Nuts and Allergy to Multiple Tree Nuts

Subsequent Reactions

Resolution of Peanut Allergy

Resolution of Tree Nut Allergy

Peanut and Tree Nut Allergens

Peanut

Case Study

Tree Nut Allergens

Hazelnut (Corylus avellana)

Walnut (Juglans regia) and Pecan (Carya illinoinensis)

Cashew (Anacardium occidentale) and Pistachio (Pistacia vera)

Almond (Prunus dulcis)

Other Tree Nuts

Pathogenesis

Conclusion

References

\section{Grain and Legume Allergy}

Ito, K. (Aichi)

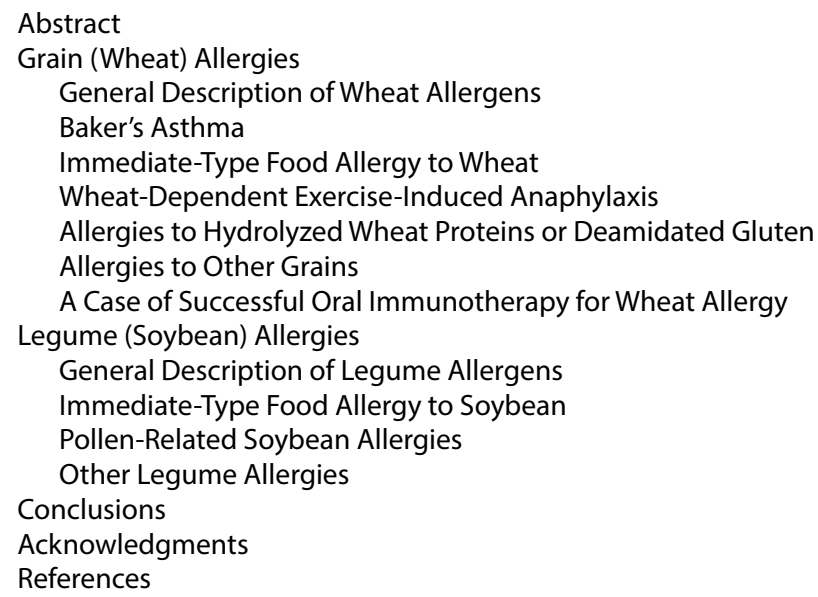

\section{Fish and Shellfish Allergy}

Thalayasingam, M.; Lee, B.-W. (Singapore)

Abstract

Introduction

Prevalence

Classification of Seafood Species

Clinical Manifestations

Fish and Shellfish Allergens

Allergenic Cross-Reactivity

Parvalbumin within the Fish Group and Frog

Within the Shellfish Group

Between Shellfish and Other Arthropods

Differential Diagnosis for Fish and Shellfish Allergy

Diagnosis

Natural History and Treatment 
162 Fruit and Vegetable Allergy

Fernández-Rivas, M. (Madrid)

162 Abstract

162 Introduction

163 Epidemiology

163 Pathogenesis: Allergen Sensitisation

166 Allergens

166 Clinical Symptoms

167 Diagnosis

168 Management

169 References

\section{Disease-Based Food Allergies}

171 Gastrointestinal Food Allergies

Heine, R.G. (Melbourne, Vic.)

171 Abstract

171 Introduction

172 Clinical Presentation

172 Food Protein-Induced Enteropathy

172 Food Protein-Induced Enterocolitis Syndrome

173 Food Protein-Induced Proctocolitis

173

References

\section{Atopic Eczema and Food Allergy}

Wassmann, A. (Hannover/Hamburg); Werfel, T. (Hannover)

181 Abstract

181 Prevalence and Natural History of Food Allergies in Atopic Eczema

182 Clinical Reaction Patterns to Foods in Patients with Atopic Eczema

183 Allergens

183 Pathogenesis of Food Allergy in Atopic Eczema

183 Epidermal Barrier Dysfunction

184 Gut Barrier Dysfunction

184 Immune Response

185 Diagnosis of Food Allergy in Atopic Eczema

186 In vitro Diagnostics

186 In vivo Diagnostics

$186 \quad$ Skin Tests

187 Oral Food Challenge

188 References 
191 Abstract

191 Introduction

192 Diagnosis

193 Laboratory Evaluation

194 Management of Anaphylaxis

194 Acute Management

196 Long-Term Management and Prevention

196 Food-Dependent Exercise-Induced Anaphylaxis

197 Summary

197 References

199 Eosinophilic Oesophagitis

Heine, R.G.; Allen, K.J. (Melbourne, Vic.)

199

199

200

200

200

201

201

202

202

203

203

203

203

203

204

204

205

205

205

205

\section{Special Topics}

209

\section{Nowak-Węgrzyn, A.; Groetch, M. (New York, N.Y.)}

\section{Abstract}

Elimination Diet in Food Allergy Diagnosis

Elimination Diet in Food Allergy Management

Loopholes in the Labeling Laws

Changing Paradigms in the Management of Milk and Egg Allergy: Baked Milk and Egg Diet

The Effects of Allergen Avoidance on Nutrition in Children

Cow's Milk Allergy

Grains

Soy, Egg, Peanut, Tree Nut, Fish and Shellfish Allergies

Nutritional Impact of Specific Food-Allergic Disorders

Feeding and Developmental Needs

Nutritional Issues in Adults with Food Allergy

Summary

References 
221 Food Allergy: Psychosocial Impact and Public Policy Implications

Sharma, H.P.; Herbert, L.J. (Washington, D.C.)

221 Abstract

221 Introduction

222 Food Allergy Knowledge and Attitudes

222 Impact of Food Allergy on Daily Life

223 Current Food Allergy-Related Public Policy

224 Implications and Future Directions

225 References

227

227

227

228

231

231

233

233

233

233

234

235

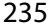

236

238

238

241

243

244

246

248

249

251

251

253

253

253

254

254

254

254

257

258

258

259

259

259

260

\section{Worldwide Food Allergy Labeling and Detection of Allergens in Processed Foods}

Taylor, S.L.; Baumert, J.L. (Lincoln, Nebr.)

Abstract

Introduction

Labeling of Allergenic Foods

Ingredient Labeling and Exemptions

Advisory Labeling

Detection of Food Allergen Residues

Quantitative Immunoassay Methods

Qualitative Immunoassay Methods

Conclusion

References

\section{The Effects of Food Allergy on Quality of Life}

DunnGalvin, A. (Cork); Dubois, A.E.J.; Flokstra-de Blok, B.M.J. (Groningen); Hourihane, J.O'B. (Cork)

Abstract

Introduction

Main Body

The Parental Perspective: Quantitative

The Child, Adolescent and Adult Perspective: Quantitative

Qualitative Studies on the Impact of Food Allergy on Health-Related Quality of Life

The Parent Perspective

Children and Teens

Discussion

Improving HRQL

Conclusion

References

Prevention of Food Allergy

Tsakok, T.; Du Toit, G.; Lack, G. (London)

Abstract

Introduction

Methodological Challenges

Onset of Sensitisation and Food Allergy

Maternal Diet (During Pregnancy and/or Breastfeeding) and the Prevention of Food Allergy

Complementary Infant Feeding and the Prevention of Food Allergy

Combined Maternal and Infant Dietary Measures and the Prevention of Food Allergy

Routes of Sensitisation

Oral Tolerance Induction

Unpasteurised Milk, Probiotics and Prebiotics

Nutritional Supplements

Conclusions

References 
263 Educational Programmes in Food Allergy

Kugler, C.; Brockow, K. (München); Ring, J. (Davos)

263 Abstract

263 Introduction

263 Individual Dietetic Treatment of Food Allergy

264 Structured Educational Group Sessions for Patients and Families with Atopic Eczema or Anaphylaxis

264 Educational Programmes for Atopic Eczema

264 Educational Programmes for the Control of Atopic Eczema in Children and Adolescents (According to 'Arbeitsgemeinschaft Neurodermitis-Schulung')

266 Educational Programmes for the Management of Atopic Eczema in Adults ('Arbeitsgemeinschaft Neurodermitis-Schulung für Erwachsene')

267 Educational Program for Anaphylaxis Patients and Families

269 References

\section{Acknowledgments}

271 Author Index

272 Subject Index 


\section{Preface}

Although food allergy was first described a long time ago, as outlined in the chapter 'Historical background, definitions, and differential diagnosis', only in recent times has it started to be recognized as one of the major allergic diseases. In the past 50 years since the discovery of IgE by Dr. K. Ishizaka, we have experienced a steep increase in the prevalence of allergic diseases. The prevalence of food allergy around the world has not yet been clarified in detail. It is quite obvious that we have faced a rapid increase in patients with food allergy in both childhood and adulthood in most developed countries in the past 3 decades. The number of publications on food allergy reached 100/year in 1971 based on a PubMed search, and it went up to 500 /year at the beginning of the 21 st century and finally to more than 1,200 /year in 2014. It is clear that food allergy research is currently a very hot topic among academics and that issues related to food allergy attract a lot of attention. We have collaborated with distinguished, world-class professionals on food allergy research and practice to put this book together. The topics are very practical and applicable to your practice. We hope that this book on food allergy will help clinicians, academics, and paramedics to better understand current food allergy practice and research.

Motohiro Ebisawa, Sagamihara Barbara K. Ballmer-Weber, Zurich Stefan Vieths, Langen Robert A. Wood, Baltimore, Md. 
